\title{
Forces and Currents in Carbon Nanostructures: Are We Imaging Atoms?
}

\author{
Martin Ondráček, ${ }^{1}$ Pablo Pou, ${ }^{2}$ Vít Rozsíval, ${ }^{1}$ Cesar González, ${ }^{3}$ Pavel Jelínek, ${ }^{1}$ and Rubén Pérez ${ }^{2, *}$ \\ ${ }^{1}$ Institute of Physics, Academy of Sciences of the Czech Republic, Cukrovarnická 10, 162 00, Prague, Czech Republic \\ ${ }^{2}$ Departamento de Física Teórica de la Materia Condensada, Universidad Autónoma de Madrid, E-28049 Madrid, Spain \\ ${ }^{3}$ Instituto de Ciencia de Materiales de Madrid (ICMM-CSIC), E-28049 Madrid, Spain
}

(Received 17 November 2010; published 25 April 2011)

\begin{abstract}
First-principles calculations show that the rich variety of image patterns found in carbon nanostructures with the atomic force and scanning tunneling microscopes can be rationalized in terms of the chemical reactivity of the tip and the distance range explored in the experiments. For weakly reactive tips, the Pauli repulsion dominates the atomic contrast and force maxima are expected on low electronic density positions as the hollow site. With reactive tips, the interaction is strong enough to change locally the hybridization of the carbon atoms, making it possible to observe atomic resolution in both the attractive and the repulsive regime although with inverted contrast. Regarding STM images, we show that in the near-contact regime, due to current saturation, bright spots correspond to hollow positions instead of atomic sites, providing an explanation for the most common hexagonal pattern found in the experiments.
\end{abstract}

DOI: 10.1103/PhysRevLett.106.176101

PACS numbers: 68.37.Ps, 61.46.-w, 68.37.Ef

Fullerenes, nanotubes, graphene, and carbon nanoribbons are among the most promising materials for nanotechnological applications due to their unique mechanical and electronic properties [1,2]. Turning these expectations into real-world devices requires tools like the STM and the atomic force microscope (AFM) with true atomic resolution (FM-AFM) [3] to visualize, characterize, and manipulate these materials at the atomic scale. However, in spite of the apparent simplicity of the common honeycomb structure and the long-standing experimental research effort, we do not know yet something as fundamental as whether the maxima in the scanning probe microscopy images correspond to atoms or to the hollow sites [4-7]. Here, we use first-principles calculations to show that the rich variety of AFM and STM image patterns found in carbon nanostructures can be rationalized in terms of the chemical reactivity of the tip and the distance range explored in the experiments.

The first STM images of the graphite(0001) surface did not display the expected honeycomb pattern but a hexagonal arrangement of bright spots (topographic maxima; see Fig. 1 in [8]) [9-11]. The accepted interpretation relies on a subtle electronic effect [12]. The Bernal stacking makes the two surface atoms inequivalent. $C_{\alpha}$ atoms, with a nearest neighbor right below in the second layer, do not contribute significantly to the density of states close to the Fermi level, and only the $C_{\beta}$ atoms are imaged as bright spots at low bias voltages. Although a honeycomb pattern should be recovered for larger biases, the experimental evidence accumulated over the past 25 years [9-13] shows that STM images with a hexagonal pattern are overwhelmingly recorded over a broad range of bias voltages and distance operation conditions. Nevertheless, several groups have reported honeycomb patterns even for small bias voltage [13-17].
The FM-AFM, relying on the forces between the tip and surface, is expected not to be so sensitive to electronic effects and to reflect the real atomic structure of the surface. However, the first reported FM-AFM image on a highly oriented pyrolytic graphite(0001) surface [18] also showed a hexagonal array of bright spots. According to simulations using Lennard-Jones potentials to describe the van der Waals (vdW) interaction between the atoms in the Si tip and the sample, these attractive force maxima do not correspond to any of the atoms but to the hollow sites [4]. Graphite was later reexamined with a new approach to the FM-AFM that uses a metallic tip attached to one of the arms of a tuning fork as the sensing element $[5,19]$. The high stiffness $(k \sim 2000 \mathrm{~N} / \mathrm{m})$ makes it possible to operate the microscope with a small oscillation amplitude. This fact, together with the use of metallic tips, allows the simultaneous measurement of forces and tunneling currents as a function of the tip-sample distance. This spectroscopy study revealed that, contrary to the expected power law behavior for a vdW interaction, the force varies exponentially in the near-contact region. Moreover, constant-height AFM and STM images differ substantially depending on the distance and bias voltage. While STM images usually show a hexagonal array of bright protrusions, identified as the $C_{\beta}$ atoms, AFM ones evolve from a hexagonal-like pattern with maximum attraction on the $C_{\alpha}$ atoms to a honeycomb pattern where the six carbon atoms are imaged in the repulsive regime (Fig. 1 in [8]). Recent AFM studies using also very low oscillation amplitudes to enhance the sensitivity to short-range interactions are contradictory, reporting images with different symmetries. The $3 \mathrm{D}$ force maps recorded with a tuning fork at $6 \mathrm{~K}$ with an outstanding accuracy [7] confirm the very small differences in force among the different sites and show a hexagonal arrangement of bright spots for all the distances. 
On the other hand, in an AFM study at room temperature using higher flexural modes to operate with a similarly small amplitude [20], all of the carbon atoms were imaged.

STM and AFM experiments on graphite are difficult to interpret, as carbon planes can be easily pulled out by the interaction with the tip, resulting in local contact for relatively far distances. Single wall carbon nanotubes (SWCNTs), much stiffer in the normal direction, offer an excellent alternative to determine the variation of forces and currents with distance. FM-AFM images with atomic resolution on SWCNTs have been reported using semiconductor tips $[6,21]$. Clear atomic contrast is reached only at small tip-sample distances. Force spectroscopy data on different sites show maximum attractive forces that are a factor of 10 smaller than those typical for semiconductor surfaces [6]. Therefore, a vdW tip-CNT interaction has been assumed, and the bright spots in the images have been interpreted as hollow sites [6].

Understanding this puzzling situation requires a theoretical analysis that identifies unambiguously the interaction responsible for the atomic contrast in low-dimensional carbon materials and that can reconcile the apparently contradictory results. To that end, we have carried out an extensive set of first-principles calculations to map out the tip-sample interaction and the electronic currents between several tip apexes with different chemical reactivity and both a SWCNT and a graphite(0001) surface. For an accurate representation of the interaction, we combine density functional theory calculations (VASP [22,23]) using the Perdew-Burke-Ernzerhof (PBE) exchange-correlation functional [24] for the short-range (SR) chemical force [25-27] with two semiempirical atomistic approaches for the vdW interaction [28,29] (see [8] for details). While continuous approaches can model the long-range vdW interaction between the macroscopic parts of the tip and sample, a more accurate atomistic scheme is needed to describe the interaction between the closest atoms in the tip and sample, particularly in this case where experiments seem to suggest that $\mathrm{vdW}$ interaction competes and even dominates over the SR-chemical force in the distance range where atomic contrast is observed.

First of all, we tackle the question about the nature of the interaction that provides the atomic contrast. We have chosen a Si tip with a dimer at the apex-this one gives the lowest maximum forces $(\sim 1 \mathrm{nN})$ on semiconductor surfaces [30] - and a $(17,0)$ SWCNT. Force versus distance curves for the tip-SWCNT interaction on top of a carbon atom and on the hollow site are shown in Fig. 1. Calculations including only PBE (triangles, green) show almost no attraction (compared with maximum attractive forces of $\sim 0.15 \mathrm{nN}$ in the experiments [6]). Differences between hollow and top sites appear only for very small distances, close to the repulsive regime. The addition of the attractive $\mathrm{vdW}$ interaction (circles, red) brings the total PBE + vdW force (black squares) to a good agreement

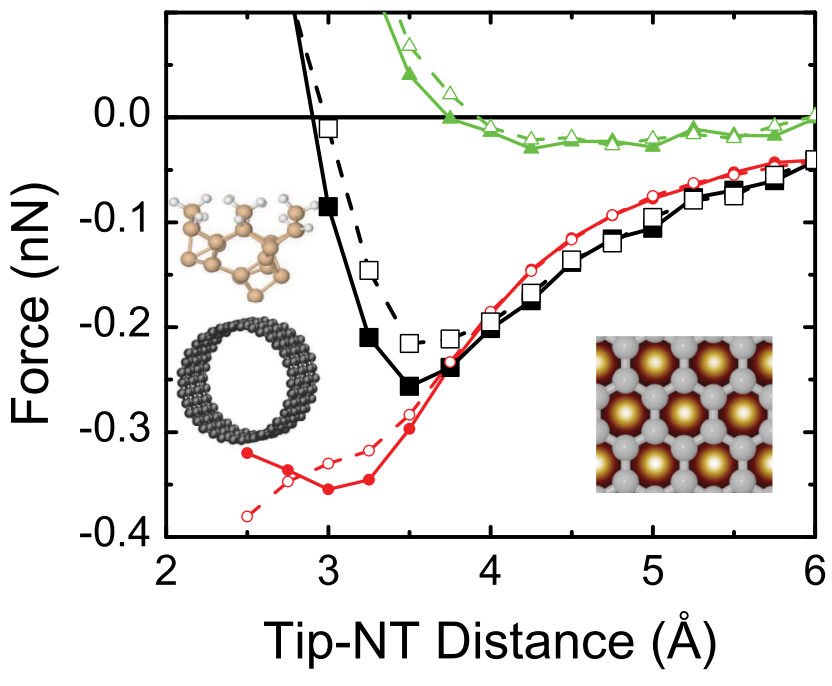

FIG. 1 (color online). Total force (black) for a dimerterminated Si tip and a $(17,0)$ SWCNT. Open (solid) symbols correspond to the tip on a carbon atom (hollow site). The vdW interaction (red, circles) gives a common attractive background that sets the absolute force values, but the short-range interaction (green, triangles) provides the atomic scale contrast in the nearcontact regime, with force maxima on the hollow site (inset).

with the experiments. The vdW interaction provides a common attractive background that dominates the absolute values of the force but does not yield itself atomic contrast: Differences over hollow and top sites for distances smaller than the force maximum do contribute to enhance the atomic resolution, but they are a consequence of the different atomic relaxations induced by the repulsive part of the SR-chemical interaction. This interaction, essentially the Pauli repulsion between electronic clouds, is larger on the areas of higher electron density (e.g., on top of the carbon atoms). Consequently, the dimer Si apex yields larger attractive forces on hollow sites, which thus correspond to the bright spots in the experimental FM-AFM images [21].

We have explored the generality of the results discussed above by calculating the interaction of the same SWCNT with other three tip apexes that span a broad range of different chemical reactivities and tip-sample interaction strengths: an oxygen-terminated Si tip [Fig. 2(a), squares], a clean Si tip with the (111) orientation [H3 Si tip, Fig. 2(a), hexagons], and a metallic $W$ tip [Fig. 2(b)]. The oxygen-terminated $\mathrm{Si}$ tip behaves almost identically to the dimer tip: The maximum attractive force is $\sim 0.3 \mathrm{nN}$ (again very close to the experiments on SWCNTs measured with silicon tips [6]), and the atomic contrast is produced by the SR electronic interaction (see Fig. 2 in [8]), resulting in force maxima on the hollow sites. The H3-(111) Si tip provides a completely different scenario. This tip [Fig. 2(a), hexagons] yields larger forces on both sites that are almost identical up to a distance of $3.5 \AA$. From this point, the interaction decreases on the hollow site 

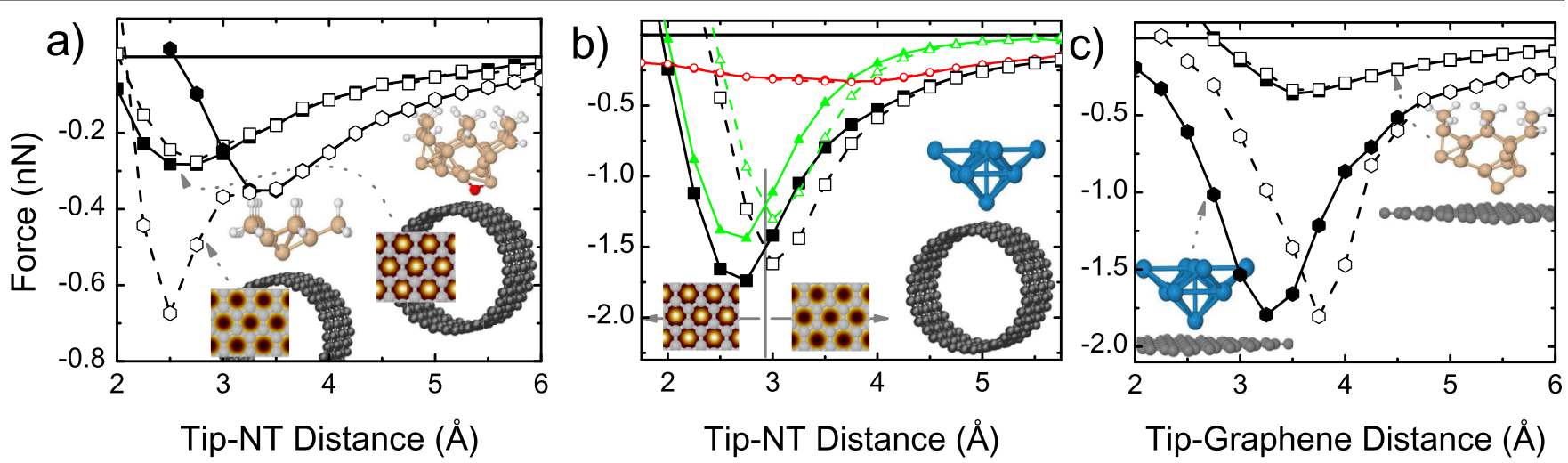

FIG. 2 (color online). Forces on a SWCNT and on graphite(1000) with tips of different chemical reactivity. The color code and symbols are as in Fig. 1. Reactive tips [ $W$ tip, H3-Si tip in (a)] change locally the hybridization and provide force maxima on the atoms.

and grows significantly on the top site, reaching a maximum of $\sim 0.7 \mathrm{nN}$. The stronger interaction is related to the higher reactivity of this tip and is consistent with calculations on other surfaces $[27,30]$. The high reactivity of the dangling bond in the outermost apex atom makes a change in the hybridization of the carbon atom on the nanotube energetically favorable, resulting in the formation of a chemical bond. The tip reactivity, that brings a small but distinctive attractive interaction on the top site, thus changes the nature of the atomic contrast, with the force maxima now on the carbon atoms.

The $W$ tip, the most reactive of those considered, confirms this trend [Fig. 2(b)]. A strong SR attractive interaction yields maximum forces of $\sim 1.5 \mathrm{nN}$ on the carbon atoms that compare well with previous calculations using a blunter tip [31]. Beyond that point, the interaction on the top site decreases due to the strong Pauli repulsion, and there is a crossing of the force curves that results in force maxima on the hollow sites. Thus, our results predict a reversal of the FM-AFM image contrast for very close distances when metallic tips are used.

The link between tip reactivity and image contrast found for the SWCNTs also holds for graphite. We have performed calculations for the two extreme cases, the Si-dimer and $W$ tips, interacting with a flat graphene sheet and with a bilayer with the $A B$ stacking [Fig. 2(c)]. The weakly reactive Si tip yields very small SR attractive force and the vdW contribution dominates. The contrast between the hollow and top sites appears in the repulsive region and can be understood in terms of the Pauli repulsion (see Fig. 3 in [8]). Therefore, hollow positions, with smaller electronic densities, yield larger attractive forces. On the contrary, with the $W$ apex or other metallic tips [8], the SR attractive forces dominate and give rise to atomic contrast in the attractive regime with force maxima on the carbon atoms. At closer distances, we enter the repulsive part of the interaction for the top site and a crossing between force curves takes place, leading to an inversion of the image contrast. These changes in the topography images (from honeycomb to hexagonal) with the tip-sample distance have been experimentally observed in graphite [7,19]. We expect the same contrast inversion on materials with similarly high surface atomic densities like closed-pack metallic surfaces [32].

The pattern that emerges from these calculations is that the tip reactivity plays a crucial role in the determination of the nature of the atomic contrast. For very reactive tips, particularly metallic ones, the SR attractive interaction dominates the atomic contrast and gives a honeycomb contrast with maximal forces on the atoms. With more inert tips, including the $\mathrm{Si}$-dimer and the O-terminated tip, the total force is attractive mainly due to the $\mathrm{vdW}$ contribution. SR interactions are essentially repulsive, and force maxima correspond to the positions with smaller electronic density like the hollow sites, resulting in a hexagonal pattern. Our study implies that the optimal choice for imaging carbon nanostructures, including organic molecules with a polycyclic aromatic core, is a chemically inert tip. A recent FM-AFM study of pentacene deposited on an alkali halide ultrathin film has shown the potential of this approach [33] using a closed-shell CO molecule attached to a metallic tip as the probing element. This functionalized tip yields larger attractive forces on the hollow sites, in agreement with our conclusions. More importantly, it provides an astonishing resolution in constant-height images probing the SR repulsive regime, where it is able to map even the covalent bonds between the carbon atoms.

Having shown that the AFM contrast with metallic tips does depend on the tip-sample distance, the question that naturally arises is whether the transport properties are also affected and conflicting STM images on graphite could be explained in terms of the operation conditions. We have carried out simulations of the current between a $W$ tip and a graphene bilayer. We map the first-principles Hamiltonian in a local orbital basis [34] and use a nonequilibrium Green's function formalism that includes the multiple scattering effects to all orders [8,32]. The critical importance of these effects in the evolution of the contrast can be clearly seen in the constant-height profiles (Fig. 3) for a bias 

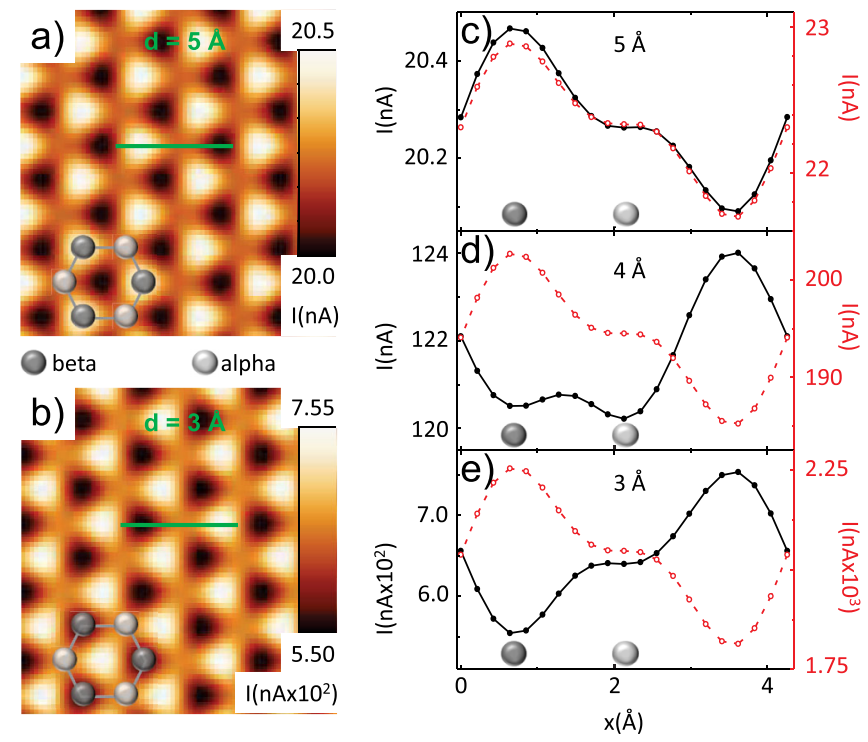

FIG. 3 (color online). Constant-height STM images on graphite(0001) (bias $=-300 \mathrm{mV}$ ), including multiple scattering effects, for a tip-surface distance of (a) 5 and (b) $3 \AA$. Both images display a hexagonal pattern but with maxima on either $C_{\beta}$ atoms or hollow sites. (c)-(e) Line profiles at three distances show that multiple scattering contributions, not included in the common perturbative approach (red line), are needed to capture the contrast inversion found in the exact solution (black line).

voltage of $-300 \mathrm{mV}$ (filled states). At typical tunneling distances, we reproduce the classical result: The $C_{\beta}$ sites, yielding larger currents, correspond to the bright spots in the STM images. In the near-contact regime, multiple scattering effects become relevant. The current on top of the carbon atoms deviates from the exponential behavior and saturates $[8,32]$. Therefore, at distances smaller than $4 \AA$, the hollow sites become the brighter spots, leading to a contrast inversion that cannot be captured by the standard perturbative approaches considered so far $[5,12,13]$. Atomic relaxations induced by the tip-sample interaction, not included in our STM calculations, could enhance the top-hollow STM corrugation in the attractive interaction regime favoring honeycomblike patterns [8].

Our results confirm that tips with very different chemical reactivity can provide atomic resolution. However, inert tips are possibly the best choice for imaging carbon nanostructures and organic molecules as they minimize possible distortions or even removal of the adsorbed material. Under these conditions, although the short-range chemical interaction is responsible for the atomic contrast, $\mathrm{vdW}$ interactions control the absolute force scale. Force spectroscopy measurements thus offer the possibility to quantify these interactions and test the performance of novel theoretical descriptions [35] of the subtle nonlocal correlations responsible for the vdW forces.

We thank M. Ashino for fruitful discussions. We acknowledge the support of Projects No. 202/09/0545 and
No. 204/10/0952 (GAČR), No. M100100904 (GAAV), No. MAT2008-02929-NAN, No. MAT2008-02939-E, No. MAT2008-01497, and No. CSD2007-41 (MICINN, Spain), a JAE-doc contract (CSIC), and the Ramón y Cajal Program (MICINN). Computer time was provided by the Spanish Supercomputing Network (RES) and by the CeSViMa (Madrid).

*Corresponding author. ruben.perez@uam.es

[1] M. S. Dresselhaus et al., Science of Fullerenes and Carbon Nanotubes (Academic, New York, 1996).

[2] A. K. Geim and K. S. Novoselov, Nature Mater. 6, 183 (2007).

[3] S. Morita et al., Noncontact Atomic Force Microscopy (Springer-Verlag, Berlin, 2002).

[4] H. Hölscher et al., Phys. Rev. B 62, 6967 (2000).

[5] S. Hembacher et al., Proc. Natl. Acad. Sci. U.S.A. 100, 12539 (2003).

[6] M. Ashino et al., Phys. Rev. Lett. 93, 136101 (2004).

[7] B. J. Albers et al., Nature Nanotech. 4, 307 (2009).

[8] See supplemental material at http://link.aps.org/ supplemental/10.1103/PhysRevLett.106.176101 for additional information.

[9] G. Binnig et al., Europhys. Lett. 1, 31 (1986).

[10] S.-I. Park and C.F. Quate, Appl. Phys. Lett. 48, 112 (1986).

[11] H. J. Mamin et al., Phys. Rev. B 34, 9015 (1986).

[12] D. Tománek et al., Phys. Rev. B 35, 7790 (1987).

[13] E. Cisternas et al., Phys. Rev. B 79, 205431 (2009).

[14] H. A. Mizes, S. I. Park, and W. A. Harrison, Phys. Rev. B 36, 4491 (1987).

[15] P. J. Ouseph et al., Phys. Lett. A 205, 65 (1995).

[16] J. I. Paredes et al., Carbon 39, 476 (2001).

[17] Y. Wang et al., Surf. Sci. 600, 729 (2006).

[18] W. Allers et al., Appl. Surf. Sci. 140, 247 (1999).

[19] S. Hembacher et al., Phys. Rev. Lett. 94, 056101 (2005).

[20] S. Kawai and H. Kawakatsu, Phys. Rev. B 79, 115440 (2009).

[21] M. Ashino et al., Nanotechnology 16, S134 (2005).

[22] G. Kresse and J. Furthmuller, Phys. Rev. B 54, 11169 (1996).

[23] G. Kresse and D. Joubert, Phys. Rev. B 59, 1758 (1999).

[24] J. P. Perdew, K. Burke, and M. Ernzerhof, Phys. Rev. Lett. 77, 3865 (1996).

[25] R. García and R. Pérez, Surf. Sci. Rep. 47, 197 (2002).

[26] M. A. Lantz et al., Science 291, 2580 (2001).

[27] Y. Sugimoto et al., Nature (London) 446, 64 (2007).

[28] S. Grimme, J. Comput. Chem. 25, 1463 (2004).

[29] M. Elstner et al., J. Chem. Phys. 114, 5149 (2001).

[30] P. Pou et al., Nanotechnology 20, 264015 (2009).

[31] C. González et al., Phys. Rev. Lett. 102, 106801 (2009).

[32] J. M. Blanco et al., Phys. Rev. B 70, 085405 (2004).

[33] L. Gross et al., Science 325, 1110 (2009).

[34] P. Jelínek et al., Phys. Rev. B 71, 235101 (2005).

[35] A. Tkatchenko et al., MRS Bull. 35, 435 (2010). 